\title{
Deconfinement phase transitions in external fields
}

\section{Paolo Cea*}

Dipartimento Interateneo di Fisica, Università di Bari and INFN - Sezione di Bari, I-70126 Bari, Italy

E-mail: Paolo.Cea@ba.infn.it

\section{Leonardo Cosmai}

INFN - Sezione di Bari, I-70126 Bari, Italy

E-mail: Leonardo. Cosmai@ba.infn.it

\begin{abstract}
We compare vacuum dynamics of abelian versus non abelian lattice gauge theories in presence of external fields. We find that the deconfinement temperature of non abelian theories depends on the strength of a constant abelian chromomagnetic field end eventually goes to zero. On the contrary such an effect is not shared by abelian theories. We argue on the relevance of this result to understand the QCD vacuum.
\end{abstract}

XXIIIrd International Symposium on Lattice Field Theory

25-30 July 2005

Trinity College, Dublin, Ireland

${ }^{*}$ Speaker. 


\section{Introduction}

A fundamental problem in high energy physics is to understand color confinement. Indeed, the mechanism that leads to color confinement remains an open question despite intense lattice studies for nearly three decades (for recent reviews on confinement see [1-3]). In order to investigate vacuum structure of lattice gauge theories both at zero and finite temperature, we introduced a lattice gauge invariant effective action $\Gamma\left[\vec{A}^{\text {ext }}\right]$ for gauge systems in external static background fields $\vec{A}^{\text {ext }}$ [4]. If we now consider the gauge theory at finite temperature $T=1 /\left(a L_{t}\right)$ in presence of an external background field, the relevant quantity turns out to be the free energy functional $\mathscr{F}\left[\vec{A}^{\text {ext }}\right]$. We are interested in vacuum dynamics of $\mathrm{U}(1), \mathrm{SU}(2)$, and $\mathrm{SU}(3)$ lattice gauge theories under the influence of an abelian chromomagnetic background field. In our previous studies we found that in $\mathrm{SU}(3)$ the deconfinement temperature depends on the strength of an applied external constant abelian chromomagnetic field. This is at variance of abelian magnetic monopoles where the abelian monopole background fields do not modify the deconfinement temperature. We would like to ascertain if the dependence of the deconfinement temperature on the strength of an applied external constant abelian chromomagnetic field is a peculiar feature of non abelian gauge theories. Since our lattice has the topology of a torus, the magnetic field turns out to be quantized $a^{2} \frac{g H}{2}=\frac{2 \pi}{L_{1}} n_{\mathrm{ext}}$, $n_{\text {ext }}$ integer. Moreover, since the free energy functional $\mathscr{F}\left[\vec{A}^{\mathrm{ext}}\right]$ is invariant for time independent gauge transformations of the background field $\vec{A}^{\text {ext }}$, it follows that for a constant background field the free energy $F\left[\vec{A}^{\text {ext }}\right]$ is proportional to the spatial volume $V=L_{s}^{3}$, and the relevant quantity is the density of free energy $f\left[\vec{A}^{\mathrm{ext}}\right]=\frac{1}{V} F\left[\vec{A}^{\mathrm{ext}}\right]$. We evaluate by numerical simulations $f^{\prime}\left[\vec{A}^{\mathrm{ext}}\right]$ the derivative with respect to the coupling $\beta$ of the free energy density $f\left[\vec{A}^{\text {ext }}\right]$ at fixed external field strength $g H$.

\section{2. $(3+1)$ dimensions}

In this section we report results obtained (see also Ref. [5]) in studying the finite temperature phase transition of lattice gauge theories $\mathrm{SU}(3)$ and $\mathrm{SU}(2)$ in (3+1)-dimensions, in presence of a constant abelian chromomagnetic background field.

\subsection{SU(3)}

As is well known, the pure $\mathrm{SU}(3)$ gauge system undergoes a deconfinement phase transition at a given critical temperature. Our aim is to study the possible dependence of the critical temperature from the strength of the applied field. The critical coupling $\beta_{c}$ can be evaluated by measuring $f^{\prime}\left[\vec{A}^{\text {ext }}\right]$, the derivative of the free energy density with respect to $\beta$, as a function of $\beta$. Indeed we found that $f^{\prime}\left[\vec{A}^{\text {ext }}\right]$ displays a peak in the critical region where it can be parameterized as $\frac{f^{\prime}\left(\beta, L_{t}\right)}{\varepsilon_{\mathrm{ext}}^{\prime}}=\frac{a_{1}\left(L_{t}\right)}{a_{2}\left(L_{t}\right)\left[\beta-\beta^{*}\left(L_{t}\right)\right]^{2}+1}$, where we normalize to the derivative of the classical energy due to the external applied field $\varepsilon_{\text {ext }}^{\prime}=\frac{2}{3}\left[1-\cos \left(\frac{g H}{2}\right)\right]=\frac{2}{3}\left[1-\cos \left(\frac{2 \pi}{L_{1}} n_{\text {ext }}\right)\right]$. Remarkably, we have checked that the evaluation of the critical coupling $\beta^{*}\left(L_{t}\right)$ by means of $f^{\prime}\left[\vec{A}^{\text {ext }}\right]$ is consistent with the usual determination obtained through the temporal Polyakov loop susceptibility. We varied the strength of the applied external abelian chromomagnetic background field to study quantitatively the dependence of $T_{c}$ on $g H$. It is worth to note that lattice data can be reproduced by the linear 


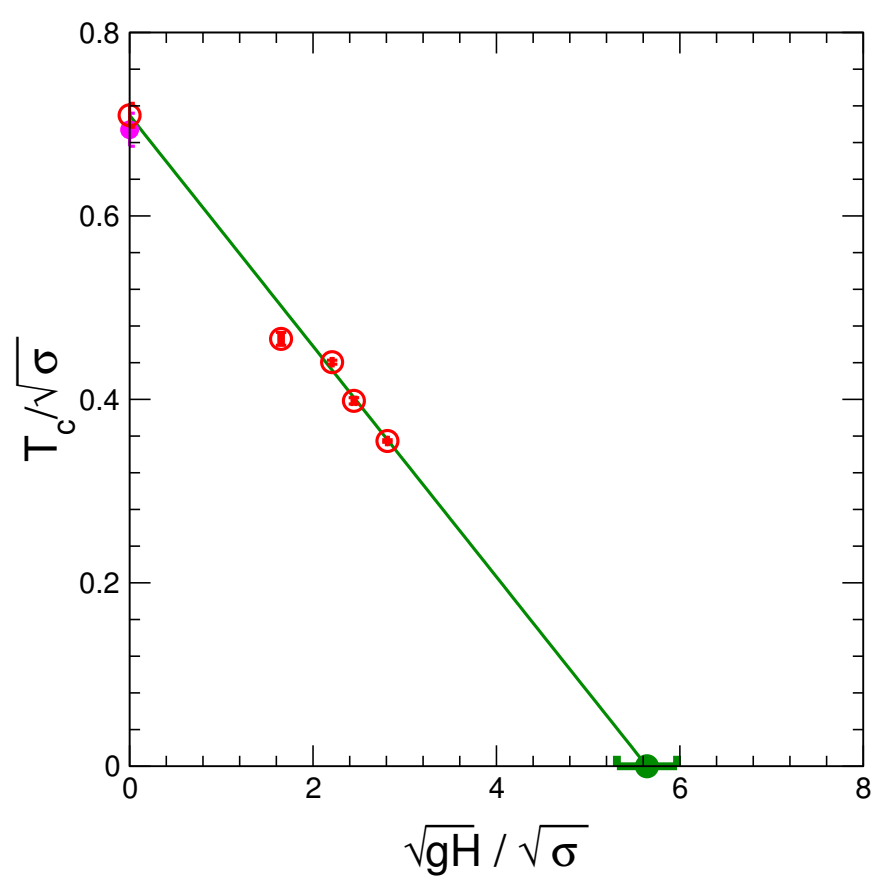

Figure 1: $\mathrm{SU}(2)$ in $(3+1)$ dimensions. The critical temperature $T_{c}$ estimated on a $64^{3} \times 8$ lattice versus the square root of the field strength $\sqrt{g H}$ in units of the string tension.

fit $\frac{T_{c}}{\sqrt{\sigma}}=\alpha \frac{\sqrt{g H}}{\sqrt{\sigma}}+\frac{T_{c}(0)}{\sqrt{\sigma}}$ with $\frac{T_{c}(0)}{\sqrt{\sigma}}=0.643(15), \alpha=-0.245(9)$. The critical field can now be expressed in units of the string tension $\frac{\sqrt{g H_{c}}}{\sqrt{\sigma}}=2.63 \pm 0.15$. Assuming $\sqrt{\sigma}=420 \mathrm{MeV}$, we find for the critical field $\sqrt{g H_{c}}=(1.104 \pm 0.063) \mathrm{GeV}$ corresponding to $g H_{c}=6.26(2) \times 10^{19}$ Gauss.

\section{$2.2 \mathrm{SU}(2)$}

We also studied the SU(2) lattice gauge theory in a constant abelian chromomagnetic field. Even in this theory the deconfinement temperature turns out to depend on the strength of the applied chromomagnetic field. We evaluated the critical coupling $\beta^{*}\left(L_{t}, n_{\mathrm{ext}}\right)$ on a $64^{3} \times 8$ lattice versus the strength of the external chromomagnetic field. As in previous section the critical coupling has been found by locating the peak of the derivative of the free energy density with respect to the gauge coupling $\beta$. In Fig. $1 T_{c} / \sqrt{\sigma}$ is plotted against $\sqrt{g H} / \sqrt{\sigma}$. As in the SU(3) case discussed in previous section, we found that the linear fit works quite well and we get $\frac{T_{c}(0)}{\sqrt{\sigma}}=0.710(13)$, $\alpha=-0.126(5)$. The value obtained for $T_{c}(0) / \sqrt{\sigma}$ is in good agreement with the value $T_{c} / \sqrt{\sigma}=$ 0.694(18), without external field, obtained in the literature. Now we can estimate the critical field in string tension units that turns out to be $\frac{\sqrt{g H_{c}}}{\sqrt{\sigma}}=5.33 \pm 0.33$. Note that the critical field $\sqrt{g H_{c}} / \sqrt{\sigma}$ is about a factor 2 greater than the $\mathrm{SU}(3)$ critical value. Our results indicate a dependence of the deconfinement temperature on the strength of a constant abelian chromomagnetic background field. On the other hand, we found that such an effect is absent for four dimensional U(1) lattice gauge theory, so that we may conclude that it is peculiar of non abelian gauge theories. In Fig. 2 we display the phase diagram for four dimensional SU(2) and SU(3) gauge theories. 


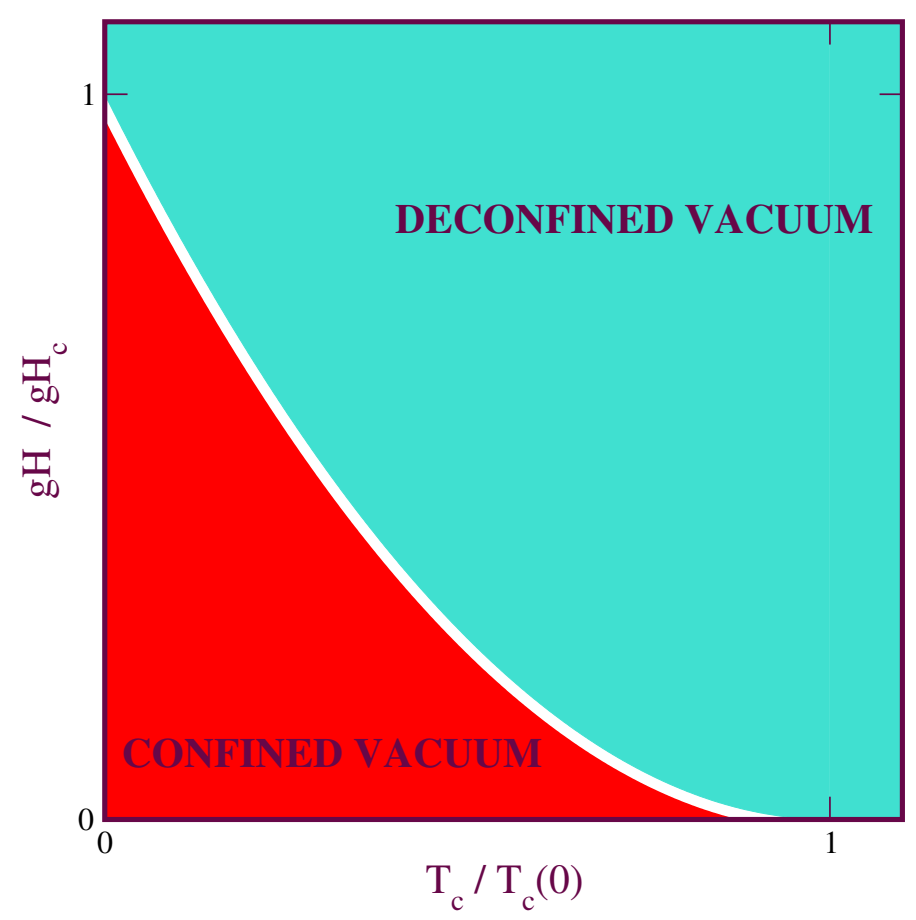

Figure 2: Phase diagram of four dimensional $S U(2)$ and $S U(3)$ gauge theories.

\section{3. $(2+1)$ dimensions}

Our numerical results for non abelian gauge theories SU(2) and SU(3) in (3+1) dimensions in presence of an abelian constant chromomagnetic background field lead us to conclude that the deconfinement temperature depends on the strength of the applied field, and eventually becomes zero for a critical value of the field strength. A natural question arises if this phenomenon, which is peculiar of non abelian gauge theories, continues to hold in $(2+1)$ dimensions. To this purpose we consider here the non abelian SU(3) lattice gauge theory to be contrasted with the abelian U(1) lattice gauge theory at finite temperature.

\subsection{SU(3)}

In this section we focus on gauge systems in $(2+1)$ dimensions. As is well known gauge theories in $(2+1)$ dimensions possess a dimensionful coupling constant, namely $g^{2}$ has dimension of mass and so provides a physical scale. In $(2+1)$ dimensions the chromomagnetic field $H^{a}$ is a (pseudo)scalar $H^{a}=\frac{1}{2} \varepsilon_{i j} F_{i j}^{a}=F_{12}^{a}$. As in the four dimensional case since we assume to have a lattice with toroidal geometry the field strength is quantized. We computed the derivative of the free energy density on a $L \times 256 \times 4$ lattice, with $L=256,512$ and several values of the external field strength parameterized by $n_{\text {ext }}$. We locate the critical coupling $\beta_{c}$ as the position of the maximum of the derivative of the free energy density at given external field strength. As for $\mathrm{SU}(3)$ in $(3+1)$ dimensions, the value of $\beta_{c}$ depends on the field strength. Using the parameterization for the string tension given in eq. (C9) of ref. [6] we are able to estimate the critical temperature $T_{c}$ in units of the string tension. We find that, as in $(3+1)$ dimensions, $T_{c} / \sqrt{\sigma}$ depends linearly on the applied 


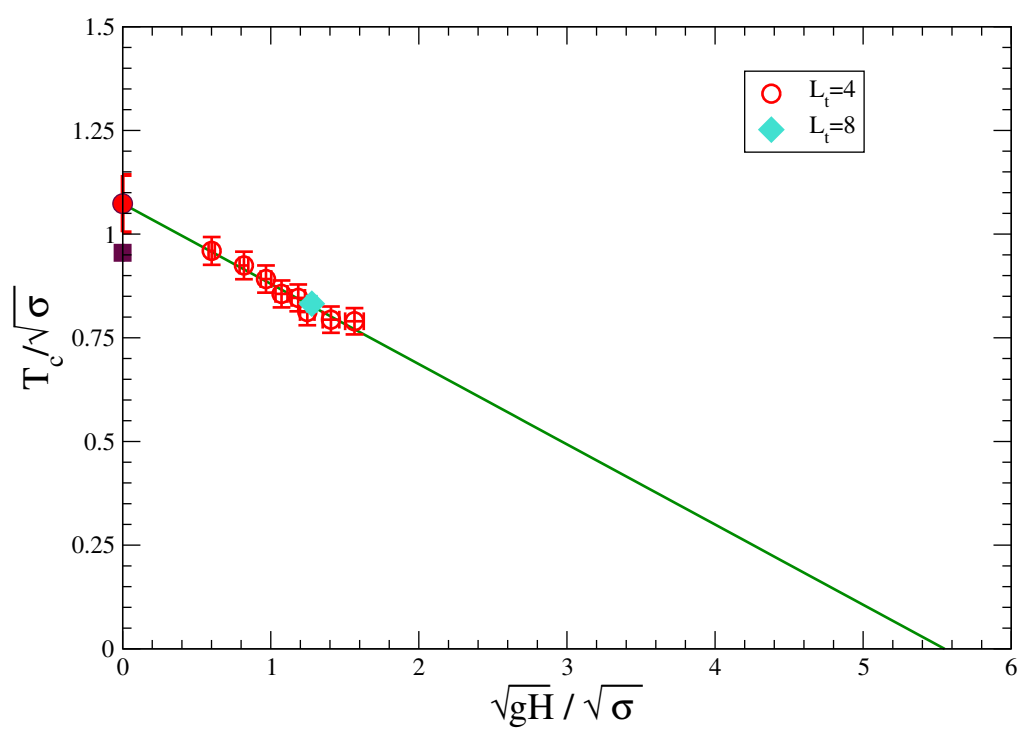

Figure 3: $\mathrm{SU}(3)$ in $(2+1)$ dimensions. The critical temperature $T_{c}$ estimated on $256^{2} \times 4,512^{2} \times 4$ and $512 \times 256 \times 8$ lattices versus the square root of the field strength $\sqrt{g H}$ in units of the string tension. Open circles refer to $L_{t}=4$, diamond to $L_{t}=8$.

field strength (see Fig. 3). The linear fit gives $\frac{T_{c}(0)}{\sqrt{\sigma}}=1.073(87), \alpha=-0.193(76)$ that implies a critical field $\sqrt{g H_{c}} / \sqrt{\sigma}=5.5 \pm 3.7$. Note that the value for $T_{c}(0) / \sqrt{\sigma}$ in the present work is in fair agreement with $T_{c} / \sqrt{\sigma}=0.972(10)$ without external field obtained in ref. [7]. To check possible finite volume effects, we performed a lattice simulation with $L_{t}=8$. The result shows that within statistical uncertainties our estimate of the critical temperature from the simulation with $L_{t}=8$ is in agreement with result at $L_{t}=4$.

\section{$3.2 \mathrm{U}(\mathbf{1})$}

In a classical paper [8] Polyakov showed that compact quantum electrodynamics in $(2+1)$ dimensions at zero temperature confines external charges for all values of the coupling. Moreover it is well ascertained that the confining mechanism is the condensation of magnetic monopoles which gives rise to a linear confining potential and a non-zero string tension. At finite temperature the gauge system undergoes a deconfinement transition which appears to be of the KosterlitzThouless type. We studied lattice U(1) gauge theory in an uniform external magnetic field. We performed numerical simulations on $512 \times 256 \times 4$ and $512 \times 64 \times 8$ lattices. To determine the critical coupling $\beta_{c}$, we measure the derivative of the free energy density. Contrary to the case of $(2+1)$ and $(3+1)$ non abelian lattice gauge theories, we do not find a dependence of the critical value of the coupling $\beta_{c}$ on the magnetic field strength. By increasing the temporal size to $L_{t}=8$ the critical coupling increases and is still independent of the external magnetic field strength. Therefore we can conclude that even in $(2+1)$ dimensional case the critical coupling does not depend on the strength of the external magnetic field as for $\mathrm{U}(1)$ lattice gauge theories in $(3+1)$ dimensions. 


\section{Conclusions}

Let us conclude this paper by recalling our main results. We probed the vacuum of $U(1)$, $\mathrm{SU}(2)$, and SU(3) l.g.t.'s by means of an external constant abelian (chromo)magnetic field. We found that, both in $(2+1)$ and $(3+1)$ dimensions: for non abelian gauge theories there is a critical field $g H_{c}$ such that for $g H>g H_{c}$ the gauge systems are in the deconfined phase (we named this effect "reversible vacuum color Meissner effect"). On the other hand we also found that for abelian gauge theories the critical coupling does not depend on the strength of the external constant magnetic field.

The dependence of the deconfinement temperature on the strength of the abelian chromomagnetic field in non abelian gauge theories could be intuitively understood by considering that strong enough chromomagnetic fields would force long range color correlations such that the gauge system gets deconfined.

On a more speculative side, one may thus imagine the confining vacuum of non abelian gauge theories as a disordered chromomagnetic condensate which confines color charges due to both the presence of a mass gap and the absence of long range color correlations, as argued by R. P. Feynman for QCD in (2+1) dimensions [9].

\section{References}

[1] G. Ripka, Dual superconductor models of color confinement, vol. 639 of Lecture Notes in Phys. Springer-Verlag, 2004.

[2] J. Greensite, The confinement problem in lattice gauge theory, Prog. Part. Nucl. Phys. 51 (2003) 1, [hep-lat/0301023].

[3] R. W. Haymaker, Confinement studies in lattice QCD, Phys. Rept. 315 (1999) 153-173, [hep-lat/9809094].

[4] P. Cea and L. Cosmai, Probing the non-perturbative dynamics of SU(2) vacuum, Phys. Rev. D60 (1999) 094506, [hep-lat/9903005].

[5] P. Cea and L. Cosmai, Color dynamics in external fields, JHEP 08 (2005) 079, [hep-lat / 050500 7].

[6] M. J. Teper, $S U(N)$ gauge theories in 2+1 dimensions, Phys. Rev. D59 (1999) 014512, [hep-lat/9804008].

[7] J. Engels et. al., A study of finite temperature gauge theory in (2+1) dimensions, Nucl. Phys. Proc. Suppl. 53 (1997) 420-422, [hep-lat/9608099].

[8] A. M. Polyakov, Quark confinement and topology of gauge groups, Nucl. Phys. B120 (1977) 429-458.

[9] R. P. Feynman, The qualitative behavior of Yang-Mills theory in (2+1)- dimensions, Nucl. Phys. B188 (1981) 479. 